\title{
Filling Polymersomes with Polymers by Peroxidase-Catalyzed Atom Transfer Radical Polymerization
}

\author{
Maria Valentina Dinu, Mariana Spulber, Kasper Renggli, Dalin Wu, \\ Christophe A. Monnier, Alke Petri-Fink, Nico Bruns*
}

Polymersomes that encapsulate a hydrophilic polymer are prepared by conducting biocatalytic atom transfer radical polymerization (ATRP) in these hollow nanostructures. To this end, ATRPase horseradish peroxidase (HRP) is encapsulated into vesicles self-assembled from poly(dimethylsiloxane)-block-poly(2-methyl-2-oxazoline) (PDMS-b-PMOXA) diblock copolymers. The vesicles are turned into nanoreactors by UV-induced permeabilization with a hydroxyalkyl phenone and used to polymerize poly(ethylene glycol) methyl ether acrylate (PEGA) by enzyme-catalyzed ATRP. As the membrane of the polymersomes is only permeable for the reagents of ATRP but not for macromolecules, the polymerization occurs inside of the vesicles and fills the polymersomes with poly(PEGA), as evidenced by ${ }^{1} \mathrm{H}$ NMR. Dynamic and static light scattering show that the vesicles transform from hollow spheres to filled spheres during polymerization. Transmission electron microscopy (TEM) and cryo-TEM imaging reveal that the polymersomes are stable under the reaction conditions. The polymer-filled nanoreactors mimic the membrane and cytosol of cells and can be useful tools to study enzymatic behavior in crowded macromolecular environments.

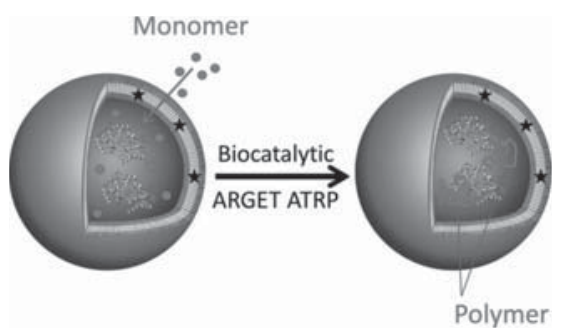

\section{Introduction}

Vesicles that are formed by self-assembly of amphiphilic blockcopolymers, so-called polymersomes, are intriguing

Dr. M. V. Dinu, Dr. M. Spulber, Dr. K. Renggli,

D. Wu, Prof. N. Bruns

Department of Chemistry, University of Basel,

Klingelbergstrasse 80, 4056 Basel, Switzerland

E-mail: nico.bruns@unifr.ch

Dr. K. Renggli

Department of Biological Engineering, Massachusetts Institute of Technology, 77 Massachusetts Avenue, Cambridge,

MA 02139, USA

C. A. Monnier, Prof. A. Petri-Fink, Prof. N. Bruns

Adolphe Merkle Institute, University of Fribourg,

Chemin des Verdiers 4, 1700 Fribourg, Switzerland nanostructures for drug delivery, ${ }^{[1]}$ for sensing, ${ }^{[2]}$ for the use as nanoreactors, ${ }^{[2,3]}$ or as mimics of cells and organelles. ${ }^{[3 b, 4]}$ They enclose a pool of water with an amphiphilic polymer bilayer and can therefore encapsulate various hydrophilic guests, such as enzymes, ${ }^{[2,3]}$ or drugs. ${ }^{[1]}$ Of particular interest for the field of biomimicry are polymer- or hydrogel-filled vesicles, because the encapsulated polymer mimics the dense cytosol or viscoelastic cytoskeleton in cells. ${ }^{[4,5]}$ This provides (polymer- and lipidbased) vesicles with an enhanced mechanical stability, ${ }^{[6]}$ improves their robustness against disassembly, ${ }^{[7]}$ and yields composite nanoparticles with distinct properties of their shell and their core. Moreover, polymers within polymersomes can influence the activity of co-encapsulated catalysts by providing a crowded, viscous reaction medium, ${ }^{[8]}$ and could stabilize encapsulated enzymes against deactivation. 
There are two main routes towards polymer-filled vesicles. The first one is to encapsulate preformed polymers, such as $\operatorname{poly}\left(N\right.$-isopropylacrylamide) (poly(NiPAAm)), ${ }^{[9]}$ poly(ethylene glycol)diacrylate, ${ }^{[10]}$ or alginate, ${ }^{[11]}$ into polymersomes, e.g., during the vesicle formation process. This can optionally be followed by a gelation step. ${ }^{[0,11]}$ However, it can become difficult to encapsulate high concentrations of polymers ${ }^{[6 c, 6 d, 7]}$ and the polymer might interfere with the self-assembly of the polymersomeforming block copolymers. ${ }^{[4]}$ A second route is to synthesize polymers in-situ inside of fully assembled polymersomes, e.g., by enzyme-catalyzed ring-opening polymerization of lactones ${ }^{[12]}$ or by UV-initiated free radical polymerization of vinyl monomers, such as NiPAAm or acrylamide. ${ }^{[6 a, 6 c, 6 d, 7,13]}$ The rationale behind this strategy is that small organic molecules, i.e., monomers, can enter vesicles that are intrinsically porous ${ }^{[12]}$ or that have been permeabilized, e.g., by the incorporation of channel proteins ${ }^{[1 a, 3 a, 14]}$ or by photo-permeabilization. ${ }^{[15]}$ As the generated macromolecules are bulky, they will be retained within the vesicles. Polymerization within vesicles (and other nanoreactors systems ${ }^{[16]}$ ) allows choosing from a wide palette of monomers, and it opens possibilities to tailor the properties of the polymer core, e.g., if it forms a film that is attached to the inside of the vesicle membrane, or if it spreads throughout the entire cavity of the vesicle. ${ }^{[7]}$ Free radical polymerizations have been successfully used for the preparation of polymer-filled ves icles. ${ }^{[6 a, 6 c, 6 d, 7,13]}$ However, given the advent of controlled/ living radical polymerizations (CRP) and the possibilities that these techniques offer with respect to the synthesis of polymers with defined molecular weight, architecture, and end-group functionality, ${ }^{[17]}$ it would be desirable to conduct CRP within polymersomes. To this end, the reaction has to be confined into the inner cavity of polymersomes. Atom transfer radical polymerization (ATRP) requires transition metal complexes as catalysts. ${ }^{[17]}$ The commonly used copper complexes are similar in size to monomers. Therefore, it is difficult to entrap these catalysts within polymersomes that are permeable for small molecules. Enzymes, on the other hand, are large enough to be retained within such polymersome nanoreactors and can maintain their activity for extended periods of time. ${ }^{[18]}$ Recently, we described that the heme proteins horseradish peroxidase (HRP) ${ }^{[19]}$ and hemoglobin ${ }^{[20]}$ are biological catalysts for ATRP. Similar results were reported for other metalloenzymes. ${ }^{[21]}$ Thus, these ATRPases are ideally suited to conduct ATRP in the inside of polymersomes. Moreover, the resulting enzyme-containing and polymer-filled polymersomes could be interesting catalytic systems for biotransformations in demanding conditions, such as nonaqueous solvents.

Here, we report the HRP-catalyzed polymerization of poly(ethylene glycol) methyl ether acrylate (PEGA) under

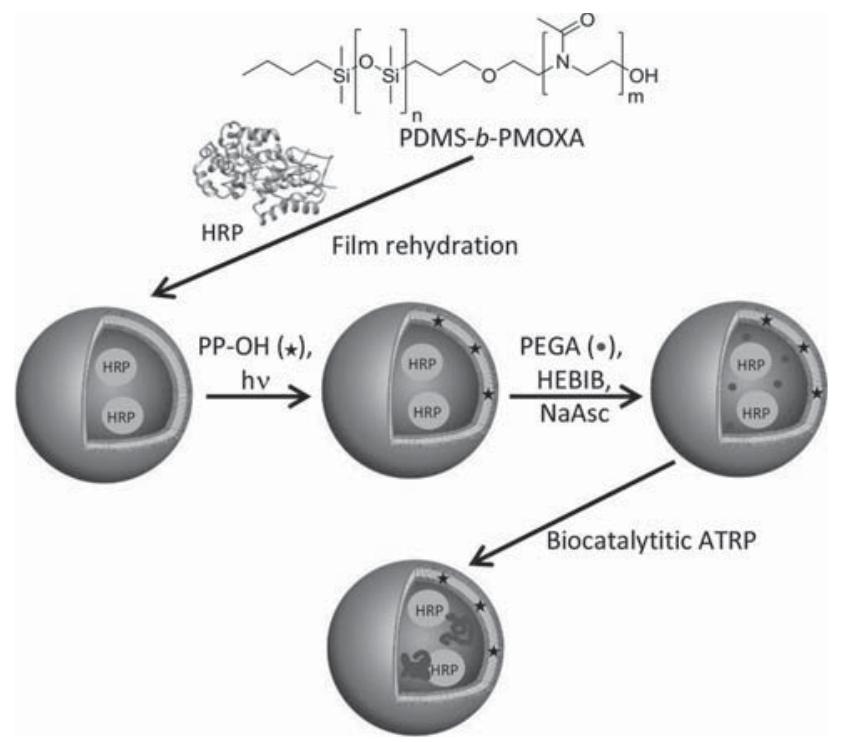

Figure 1. Filling of polymersomes with polymers by biocatalytic ATRP. Self-assembly of amphiphilic block copolymers in the presence of HRP yielded enzyme-loaded vesicles, which were subsequently permeabilized by photoreaction with an $\alpha$-hydroxyalkylphenone. Polymerization reagents (monomer PEGA, ATRP-initiator HEBIB, and reducing agent NaAsc) diffused into the vesicles where the enzyme catalyzed ATRP. As the polymersome membrane was only permeable for small molecules but not for macromolecules, the formed poly(PEGA) stayed within the polymersome and filled it.

conditions of activators regenerated by electron-transfer (ARGET) ATRP within the lumen of polymersome nanoreactors (Figure 1). Polymersomes were self-assembled from poly(dimethylsiloxane)-block-poly(2-methyl-2-oxazoline) (PDMS- $b$-PMOXA) diblock copolymers in the presence of HRP so that the enzyme was encapsulated into the polymersomes during the process of vesicle formation. Then, the membrane of the polymersomes was rendered permeable for small organic molecules by photoreaction with 2-hydroxy-4'-2-(hydroxyethoxy)-2methylpropiophenone (PP-OH) according to our previously published protocol. ${ }^{[15 a]}$ The reagents of the polymerization, i.e., initiator, reducing agent, and monomer, were added to solutions of these permeabilized polymersomes and converted by the enzyme into hydrophilic polymers that filled the interior of the polymersomes.

\section{Experimental Section}

\subsection{Materials}

The synthesis and characterization of hydroxyl end-capped PDMS $_{65}$-b-PMOXA $14\left(M_{\mathrm{n}}=6180 \mathrm{~g} \mathrm{~mol}^{-1}\right)$ was published previously. ${ }^{[22]} \mathrm{HRP}$ (highly stabilized, essentially salt-free, lyophilized powder, 200-300 units mg $^{-1}$ solid), PP-OH, 2-hydroxyethyl-2-bromoisobutyrate (HEBIB), sodium ascorbate (NaAsc), ethanol, 
tetrahydrofuran (THF; GPC grade), and deuterated chloroform $\left(\mathrm{CDCl}_{3}\right)$ were obtained from Sigma-Aldrich and were used as is. PEGA with a number-average molecular weight $M_{n}$ of $480 \mathrm{~g} \mathrm{~mol}^{-1}$ was purchased from Sigma-Aldrich and passed over neutral aluminum oxide prior to use in order to remove the inhibitor. Dulbecco's phosphate-buffered saline solution (PBS) $\left(134 \times 10^{-3} \mathrm{M} \mathrm{NaCl}, 2.7 \times 10^{-3} \mathrm{M} \mathrm{KCl}, 8.1 \times 10^{-3} \mathrm{M} \mathrm{Na}_{2} \mathrm{HPO}_{4}\right.$, $\left.1.9 \times 10^{-3} \mathrm{M} \mathrm{KH}_{2} \mathrm{PO}_{4}\right)$ at $\mathrm{pH} 7.4$ was purchased from Sigma-Aldrich.

\subsection{Preparation of Polymersome Nanoreactors}

A solution of PDMS- $b$-PMOXA ( $\left.5 \mathrm{mg} \mathrm{mL}^{-1}\right)$ in ethanol $(2 \mathrm{~mL})$ was slowly evaporated to dryness in a $5 \mathrm{~mL}$ round-bottom flask at reduced pressure using a rotary vacuum evaporator. A polymer film formed on the wall of the flask. It was rehydrated for $24 \mathrm{~h}$ at room temperature under magnetic stirring with $2 \mathrm{~mL}$ of an HRP solution $\left(2 \mathrm{mg} \mathrm{mL}^{-1}\right)$ in PBS to form enzyme-loaded vesicles. The experimental procedures for the removal of free, i.e., nonencapsulated enzyme, and for the photoreaction-induced permeabilization of the polymersome membrane with $\mathrm{PP}-\mathrm{OH}$ have been reported previously. ${ }^{[15 a]}$ A PP-OH concentration of $1 \mathrm{mg} \mathrm{mL}^{-1}$ was used. The permeabilized HRP-loaded polymersomes were purified by size-exclusion chromatography (SEC) as described previously. ${ }^{[15 a]}$

\subsection{Polymerization with Free and Encapsulated HRP}

Polymerizations were carried out in PBS with free, i.e., nonencapsulated HRP and with polymersome-encapsulated HRP, respectively. The initiator HEBIB $(8.45 \mu \mathrm{L}, 12.3 \mathrm{mg}, 0.058 \mathrm{mmol})$ and the monomer PEGA $(2120 \mathrm{mg}, 4.418 \mathrm{mmol})$ were weighed into a two-neck, round-bottom flask and dissolved in $6 \mathrm{~mL}$ PBS buffer. The solution was purged for 45 min with argon. The reducing agent NaAsc (90 mg, $0.511 \mathrm{mmol}$ ) was dissolved in $3 \mathrm{~mL}$ deoxygenated PBS buffer in a two-neck, round-bottom flask under an argon atmosphere. $2 \mathrm{~mL}$ of $0.2 \mathrm{mg} \mathrm{mL}^{-1}$ free HRP, or $0.2 \mathrm{mg} \mathrm{mL}^{-1}$ polymersome-encapsulated HRP (as determined by UV-vis spectroscopy) was purged with argon for $30 \mathrm{~min}$. The polymerization was started by transferring $2 \mathrm{~mL}$ of free HRP solution or $2 \mathrm{~mL}$ polymersome-encapsulated HRP and $2 \mathrm{~mL}$ of NaAsc solution to the initiator/monomer mixture. The reactions were stirred at room temperature for $24 \mathrm{~h}$. After $24 \mathrm{~h}$ of polymerization, the reaction mixtures were exposed to ambient air and split into two samples. One sample was lyophilized for $48 \mathrm{~h}$, after that redissolved in $\mathrm{CDCl}_{3}$ and analyzed by ${ }^{1} \mathrm{H}$ NMR to determine the conversion. The other sample was purified by SEC using Sepharose 2B. The polymersome-containing fractions were taken for GPC and ${ }^{1} \mathrm{H}$ NMR analysis. GPC samples were prepared by adding $2 \mathrm{~mL}$ of THF and then $\mathrm{MgSO}_{4}$ to remove the water. Salts were filtered off, the samples were dried a second time over $\mathrm{MgSO}_{4}$ to remove the last traces of water. Finally, the sample was filtered through a Millipore Millex-FG hydrophobic PTFE $0.2 \mu \mathrm{m}$ syringe filter into a GPC vial. ${ }^{1} \mathrm{H}$ NMR samples were prepared by lyophilization, followed by redissolving $12 \mathrm{mg}$ sample in $1 \mathrm{~mL} \mathrm{CDCl}_{3}$.

Samples for light-scattering measurements were synthesized as described above and purified by SEC using Sepharose 2B. $1 \mathrm{~mL}$ of the polymersome-containing fraction was used for lightscattering experiments.
Control reactions with polymersome-encapsulated HRP that had not been permeabilized by photoreaction with $\mathrm{PP}-\mathrm{OH}$, as well with photo-permeabilized polymersomes that lacked HRP were carried out as described above.

\subsection{Methods}

Dynamic and static light-scattering (DLS, SLS) measurements of serial dilutions with polymer concentrations ranging from 0.03 to $0.5 \mathrm{mg} \mathrm{mL}^{-1}$ were performed on an ALV goniometer (Langen, Germany) that was equipped with an HeNe laser $(\lambda=632.8 \mathrm{~nm})$. Light-scattering samples were filtered (Millex-LH syringe filters, Hydrophilic, PTFE, $0.45 \mu \mathrm{m}$; Millipore) into $10 \mathrm{~mm}$ cylindrical quartz cells and measured every $10^{\circ}$ at angles between $30^{\circ}$ and $150^{\circ}$. Cells were placed in the measuring chamber that was thermostated at $293 \mathrm{~K}$. The photon intensity autocorrelation function $\mathrm{g}_{2}(\mathrm{t})$ was determined using an ALV-7004/USB correlator. The refractive index increment for PDMS- $b$-PMOXA $(\mathrm{d} n / \mathrm{d} c=0.188)$ was taken from Nardin et al. ${ }^{[23]}$ The apparent hydrodynamic radius from DLS (cumulant analysis) and the radius of gyration from SLS (Guinier analysis) were calculated according to conventional methods. ${ }^{[24]}$ Transmission electron microscopy (TEM) images were acquired on a Philips EM400 electron microscope that was operated at $80 \mathrm{kV}$. Polymersome dispersions were deposited on a carbon-coated copper grid and negatively stained with $2 \%$ uranyl acetate solution. Polymersomes were additionally visualized under native conditions by cryo-TEM. $5 \mu \mathrm{L}$ of sample solution was deposited on a carbon-coated copper grid (Lacey carbon film grids, Plano $\mathrm{GmbH}$ ). Excess liquid was carefully blotted away with filter paper (Whatman qualitative filter paper, grade 1) before plunging the sample into a liquid ethane bath cooled by liquid nitrogen. The vitrified specimens were then kept for storage in liquid nitrogen and investigated the following day. All grids were investigated with a FEI Tecnai F20 cryo-transmission electron microscope operating at $200 \mathrm{kV}$. Images were recorded under low-dose conditions with an UltraScan 1000 CCD sensor (Gatan, Inc.) with an image resolution of $2048 \times 2048$ pixels. UVvis measurements were performed on an Analytik Jena Specord 210 plus spectrometer (Jena, Germany) with a slit width of $4 \mathrm{~nm}$ in $1 \mathrm{~cm}$ quartz cuvettes (Hellma). In order to determine HRP concentrations in samples of enzyme-loaded polymersomes (before and after photoreaction with PP-OH), the Soret absorption at 403 $\mathrm{nm}$ was measured. An extinction coefficient of $0.9 \times 10^{5} \mathrm{M}^{-1} \mathrm{~cm}^{-1}$ was used. ${ }^{[15 a]}$ GPC was performed on an Agilent Technologies 1200 HPLC system instrument equipped with Polymer Laboratories/Agilent $5 \mu \mathrm{m}$ guard column and two PLgel $5 \mu \mathrm{m}$ MIXED-C GPC columns. THF was used as eluent at a flow rate of $1 \mathrm{~mL} \mathrm{~min}{ }^{-1}$ at $30{ }^{\circ} \mathrm{C}$. Signals were recorded with a refractive-index detector $\left(\lambda=658 \mathrm{~nm}, 30^{\circ} \mathrm{C}\right.$, Optilab REX interferometric refractometer; Wyatt Technology Corp.), calibrated against polystyrene standards (Agilent) and analyzed using ASTRA software from Wyatt Technology. Proton nuclear magnetic resonance $\left({ }^{1} \mathrm{H}\right.$ NMR) was used to investigate the conversion of PEGA. ${ }^{1} \mathrm{HNMR}$ spectra were recorded on a Bruker DPX-400 spectrometer in $\mathrm{CDCl}_{3}$ and processed with MestReNova software. Conversion of PEGA was calculated from baseline-corrected spectra using the integrals of the peaks at $6.41,6.15$, and $5.84 \mathrm{ppm}$ (3H of monomer, $-\mathrm{CH}=\mathrm{CH} 2$ ) and at $3.37 \mathrm{ppm}$ (3H of monomer and polymer, -OCH3) 
(Figure S1, S2, S3, and S4, Supporting Information). The peak at $3.37 \mathrm{ppm}$ is on the "tail" of the bigger peak next to it. In order to quantify the peak correctly, a baseline correction that subtracts the component coming from the tail was carried out using multipoint baseline correction in MestReNova software. The option "Pick baseline points" was used to set baseline markers at the right and left of the peak of interest, i.e., at the edges of peak integration.

\section{Results and Discussion}

For this study, a PDMS- $b$-PMOXA diblock copolymer was selected whose self-assembly properties have recently been extensively characterized. ${ }^{[22,25]}$ It robustly forms polymersomes. The PMOXA block is hydroxyl-terminated (Figure 1) and the block copolymer does not have any unsaturated functional groups. Therefore, the block copolymer will be inert in enzyme-catalyzed radical polymerizations within the vesicles. Enzyme-loaded polymersomes were prepared by self-assembly of the block copolymers in PBS ( $\mathrm{pH} 7.4$ ) containing $2 \mathrm{mg} \mathrm{mL}^{-1} \mathrm{HRP}$ using a film rehydration method. ${ }^{[26]}$ In order to remove nonencapsulated enzyme, samples were purified by size-exclusion chromatography. Then, $\mathrm{PP}-\mathrm{OH}$ was added to the polymersome-containing fraction and the sample was briefly (30 s) irradiated with UV light. The photoreaction of PP-OH with PDMS- $b$-PMOXA in the polymersome membrane results in an increased permeability for organic molecules, turning the polymersomes into nanoreactors. ${ }^{[15 a]}$ They were purified from the reaction mixture in a further size-exclusion chromatography step. The concentration of HRP in the purified polymersome solution was measured as $0.2 \mathrm{mg} \mathrm{mL}^{-1}$ by UV-vis spectroscopy, which corresponds to an encapsulation efficiency of $10 \%$ compared to the initial enzyme concentration. The size and morphology of the enzyme-containing polymersomes were determined by light scattering (LS), TEM, and cryo-TEM (Table 1, Figure 2, Figure 3). SLS and DLS allow measuring the radius of gyration $\left(R_{\mathrm{g}}\right)$ and the hydrodynamic radius $\left(R_{\mathrm{h}}\right)$ of self-assembled objects. The ratio $R_{\mathrm{g}} / R_{\mathrm{h}}$ ( $\rho$-factor) reveals their morphology. Hollow spheres result in a $\rho$-factor of 1.0, while hard spheres give $\rho=0.77 .^{[13 a, 24]}$ PDMS- $b$-PMOXA polymersomes showed a $R_{\mathrm{g}}$ of $249 \pm 4 \mathrm{~nm}$ before and $258 \pm 4 \mathrm{~nm}$ after PP-OH treatment. The $\rho$-factor was close to 1.0 for untreated polymer vesicles and for nanoreactors after photoreaction with PP-OH (Table 1), indicating the presence of vesicles. TEM images of the samples before and after photoreaction with $\mathrm{PP}-\mathrm{OH}$ show large spherical objects with diameters between 120 and $450 \mathrm{~nm}$ (Figure 2a,b). cryo-TEM revealed that these spherical structures are hollow spheres, i.e., polymersomes (Figure 3a,b). The PP-OH-treated polymersomes have a constant membrane thickness of $19.4 \pm 1.0 \mathrm{~nm}$ (average $\pm \mathrm{SD}, n=20$ ). These images and the LS data prove that the block copolymer self-assembled into polymersomes in the presence of HRP and show that the polymersomes were not harmed by the photoreaction with PP-OH. The TEM images also reveal the presence of a few (smaller) micelles in these samples. Micelles have a hydrophobic core and do not encapsulate enzymes. Thus, these micelles will have no effect on the HRP-catalyzed polymerization within polymersomes.

In order to fill polymersomes with a hydrophilic polymer, HRP-catalyzed ATRP of PEGA was conducted with the encapsulated enzyme in vesicles. To this end, the monomer, the hydrophilic initiator HEBIB and the reducing agent $\mathrm{NaAsc}$ were added to suspensions of HRP-loaded, permeabilized polymersomes; the hypothesis being that the reagents would penetrate into the polymersomes and encounter the biocatalyst therein. Indeed, polymerization of PEGA catalyzed by encapsulated HRP resulted in an overall conversion of $40 \%$ after $24 \mathrm{~h}$ of reaction, as determined by ${ }^{1} \mathrm{H}$ NMR (Table 2). The polymersomes were purified by SEC. The ${ }^{1} \mathrm{H}$ NMR spectrum of the polymersomes shows peaks that correspond to PDMS- $b$-PMOXA and to poly(PEGA) (Figure 4). Thus, the polymersomes contained poly(PEGA). GPC revealed an elution peak with a polystyrene-apparent $M_{n}$ of $3000 \mathrm{~g} \mathrm{~mol}^{-1}$ and a dispersity $(\boxplus)$ of 1.55 (Table 2, Figure S5, Supporting Information). For comparison, biocatalytic ATRP was also carried out with free, i.e., nonencapsulated HRP in the absence of polymersomes. Poly(PEGA) in a yield of $12 \%$ was obtained after $24 \mathrm{~h}$. This polymer had a polystyrene-apparent $M_{\mathrm{n}}$ of $3430 \mathrm{~g} \mathrm{~mol}^{-1}$ and a $\boxplus$ of 1.18. Thus, HRP-catalyzed ATRP can result in relative narrowly dispersed polymers. However, when carried out in the polymersome nanoreactors, the biocatalytic polymerization resulted in polymers with a higher dispersity. This could be a result of variations of enzyme content

Table 1. Light scattering data for PDMS-b-PMOXA polymersomes.

\begin{tabular}{lccc}
\hline System & $\begin{array}{c}\boldsymbol{R}_{\mathbf{g}}{ }^{\mathbf{a})} \\
{[\mathbf{n m}]}\end{array}$ & $\begin{array}{c}\boldsymbol{R}_{\mathbf{h}}{ }^{\mathbf{b})} \\
{[\mathbf{n m}]}\end{array}$ & $\rho=\boldsymbol{R}_{\mathbf{g}} / \boldsymbol{R}_{\mathbf{h}}$ \\
\hline PDMS- $b$-PMOXA(HRP) & $249 \pm 4$ & $249 \pm 2$ & 1.00 \\
PDMS- $b-$ PMOXA(HRP) + PP-OH & $256 \pm 6$ & $258 \pm 4$ & 0.99 \\
PDMS- $b-$ PMOXA(HRP) + PP-OH + ATRP & $180 \pm 5$ & $235 \pm 2$ & 0.77
\end{tabular}

a) Derived from SLS; ${ }^{\text {b) }}$ derived from DLS. 

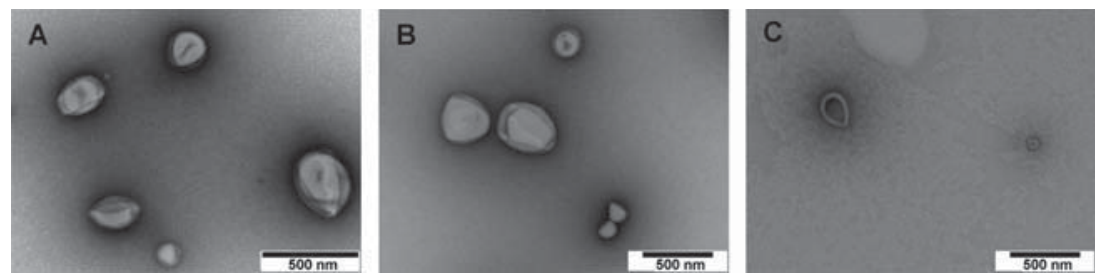

Figure 2. TEM images of HRP-loaded PMOXA- $b$-PDMS polymersomes before photoreaction with $\mathrm{PP}-\mathrm{OH}(\mathrm{A})$ and after permeabilization by photoreaction with $\mathrm{PP}-\mathrm{OH}$ (B) Poly(PEGA)-filled polymersomes obtained by HRP-catalyzed ATRP within the permeabilized polymersomes $(C)$.

between individual vesicles, since the number of propagating radicals per nanoreactors influences the dispersity of polymers. ${ }^{[16 a]}$ Enzyme encapsulation into polymersomes under similar conditions resulted in an average of 4 to 8 enzymes per polymersome, ${ }^{[27]}$ i.e., it is likely that the number of enzymes per vesicle differs between individual vesicles of the same batch.

Control reactions were carried out with HRP-filled polymersomes that had not been permeabilized by photoreaction with $\mathrm{PP}-\mathrm{OH}$, as well with photo-permeabilized polymersomes that lacked HRP. Both reactions did not yield poly(PEGA) (Table 2). The first control reaction shows that the ATRPase in the polymersomes could only be reached by reagents if the polymersome had been treated with PP-OH, i.e., native PDMS-b-PMOXA membranes were impermeable for the monomer, the initiator, and the reducing agent. The second control reaction proves that the polymerization was catalyzed by the enzyme and not caused by self-initiation of the monomer.
TEM of HRP-loaded, permeabilized PDMS- $b$-PMOXA polymersomes after $24 \mathrm{~h}$ of ATRP (Figure 2c and Figure S6, Supporting Information) showed spherical structures with diameters between 75 and $275 \mathrm{~nm}$, indicating that nanoreactors stayed intact during polymerization. Cryo-TEM images show vesicles that range in diameter from 77 to $465 \mathrm{~nm}$ (Figure $3 \mathrm{~d}-\mathrm{f}$ and Figure S7, Supporting Information). Although poly(PEGA) does not generate enough contrast to be readily visible by cryo-electron microscopy (i.e., due to the lack of heavy atoms), we observed a slightly enhanced contrast within small polymer-filled vesicles (Figure 3c-e and Figure S7, Supporting Information). Optical effects, the thickness of the vitreous ice layer or the enclosure of micelles may be contributing actors. However the observed aberrancies are atypical for any of them. It thus might be speculated that a high intrapolymersomal poly(PEGA) concentration was sufficient-especially in the small vesicles-to generate the additional contrast in the polymersomes. In addition, a defined thin layer of reduced contrast (thickness $5.9 \pm 1.3 \mathrm{~nm}$; average $\pm S D, n=20$ ) can be observed on the inside of the membrane of these small vesicles. This might be attributed to the hydrophilic PMOXA block of the block copolymers that is otherwise not observable in cryo-TEM. ${ }^{[22]}$

As the polymersomes were purified from the reaction mixture by SEC, the formed poly(PEGA) in these samples is most probably located inside of the polymersomes,

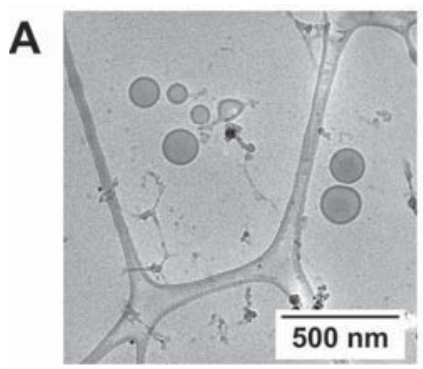

D

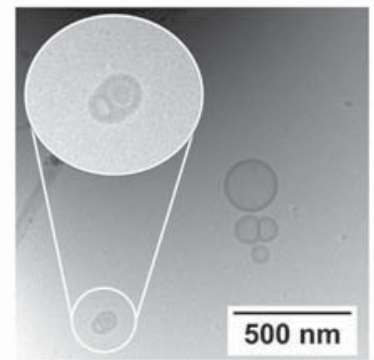

B

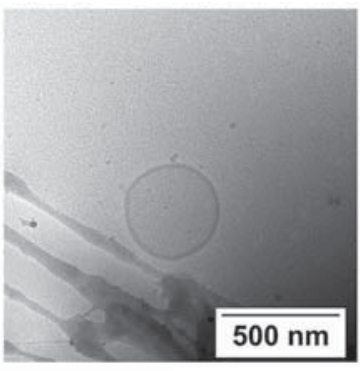

E

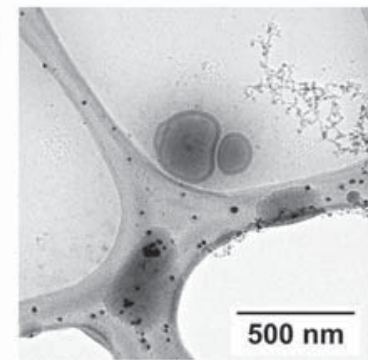

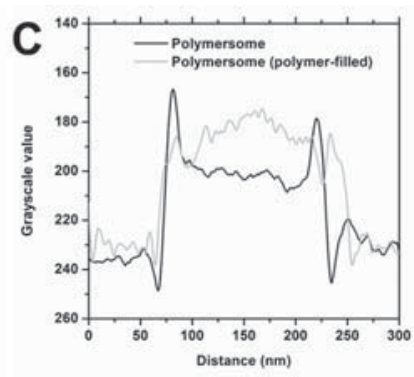

F

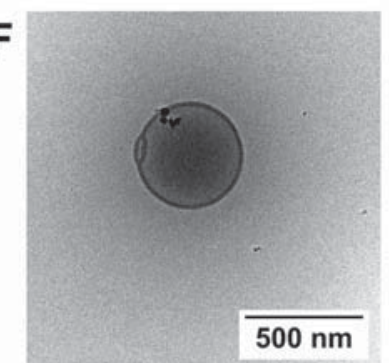

Figure 3. cryo-TEM images of HRP-loaded, photo-permeabilized PMOXA-b-PDMS polymersomes before (A, B) and after HRP-catalyzed ATRP within the polymersomes (D, E, F). Intensity profiles were plotted from representative small vesicles of both categories (C), highlighting the variation in lumen contrast between them. 
Table 2. Biocatalytic ATRP of PEGA catalyzed by HRP in solution and by HRP within polymersome nanoreactors.

\begin{tabular}{|c|c|c|c|c|}
\hline Polymerization system & $\begin{array}{c}\text { PEGA conversion }^{\mathrm{a})} \\
{[\%]}\end{array}$ & $\begin{array}{c}M_{\mathrm{n}}^{\mathrm{b})} \\
{\left[\mathrm{g} \mathrm{mol}^{-1}\right]}\end{array}$ & $\begin{array}{c}M_{\mathrm{w}}^{\mathrm{b})} \\
{\left[\mathrm{g} \mathrm{mol}^{-1}\right]}\end{array}$ & $\boldsymbol{\boxplus}$ \\
\hline Free HRP & 12 & 3430 & 4060 & 1.18 \\
\hline PDMS-b-PMOXA(HRP) + PP-OH photoreaction & 40 & 3000 & 4660 & 1.55 \\
\hline PDMS- $b$-PMOXA(HRP), no PP-OH photoreaction & 0 & - & - & - \\
\hline PDMS- $b$-PMOXA + PP-OH photoreaction & 0 & - & - & - \\
\hline
\end{tabular}

a) Calculated after a reaction time of $24 \mathrm{~h}$ from ${ }^{1} \mathrm{H}$ NMR spectra presented in Figures S1 to S5 (Supporting Information); b)from GPC curves presented in Figure S5 (Supporting Information), calibrated against PS standards.

otherwise the polymer would have been separated from the vesicles during chromatography. The presence of poly(PEGA) within the lumen of the polymersomes was proven by light scattering (Table 1). DLS yielded an hydrodynamic radius of $235 \pm 2 \mathrm{~nm}$ for PDMS- $b$-PMOXA(HRP)$\mathrm{PP}-\mathrm{OH}$ polymersomes post-polymerization, which is slightly lower than the hydrodynamic radius of the vesicles before polymerization. However, the radius of gyration (as measured by SLS) was markedly lower than the $R_{\mathrm{g}}$ of the HRP-filled polymersomes prior to ATRP. Taken together, these results reflect a shift in the polymersomes' center of mass towards the middle of the polymersomes, most likely caused by the filling of the vesicles with polymer and shrinking of the polymersomes due to the polymerization. As a result, the $\rho$-factor decreased from 0.99 to 0.77 , which corresponds to a shift in morphology from hollow spheres to filled spheres. ${ }^{[13 a, 24 a]}$ In other words, the polymerization within the nanoreactors resulted in the formation of polymersomes with a polymer core.

\section{Conclusion}

Encapsulation of enzymes into polymersomes allows confining biocatalytic reactions into the aqueous lumen of these nanoreactors. We took advantage of this concept in order to fill polymersomes with a hydrophilic polymer. To this end, HRP, an ATRPase that catalyzes ATRP, was enclosed into PDMS- $b$-PMOXA polymersomes. They were made permeable for ATRP reagents by UV-induced reaction with $\mathrm{PP}-\mathrm{OH}$. This treatment did not disturb the ability of polymersomes to retain macromolecules within their interior, i.e., polymers that were synthesized within the vesicles remained there and filled the polymersomes. Biocatalytic ATRP of PEGA yielded polymers with a relatively

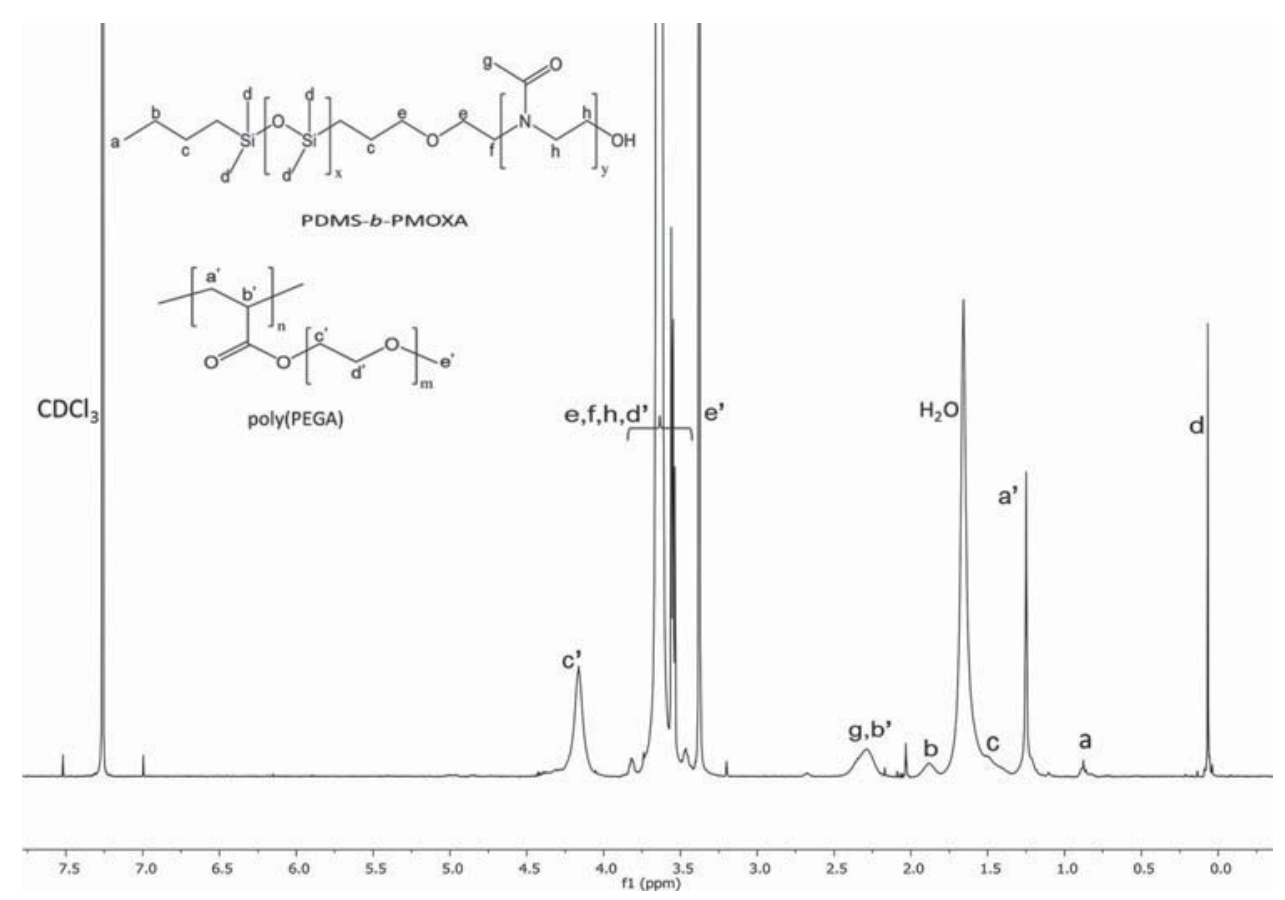

Figure 4. 'H NMR spectrum of poly(PEGA)-filled PDMS- $b$-PMOXA polymersomes purified by SEC. 
low polydispersity index. TEM and cryo-TEM revealed that polymersomes remained intact during polymerization. ${ }^{1} \mathrm{H}$ NMR of polymersomes that were purified by SEC proved the presence of poly(PEGA) in polymersomes. Light scattering showed the formation of structures with a $\rho$-factor of 0.77 , which is characteristic for filled spheres, i.e., for polymersomes with a swollen core.

Packing polymers into polymersomes via biocatalytic ATRP is a useful route to create vesicles that mimic two key aspects of cells: the cellular membrane and a viscous cytosol. Controlled radical polymerizations allow tailoring the properties of polymers in a much better way than, e.g., free radical polymerizations. Therefore, biocatalytic ATRP within polymersomes could be used to tune the properties of the bio-mimetic cytosol, e.g., by choosing the molecular weight of the formed polymer or by the synthesis of polymers with advanced architectures, such as block copolymers and star-shaped polymers. Polymercontaining polymersomes could find applications as artificial organelles and nanoreactors. In such systems, the polymer core would influence the activity and stability of encapsulated biocatalysts by providing a crowded environment, similar to the dense cytosol. Polymer-filled nanoreactors can represent model systems to study the behavior of enzymes in crowded microenvironments. Moreover, the polymer core provides a hydrophilic microenvironment that could be used as a nanoscopic reaction space for biotransformations in organic solvents.

\section{Supporting Information}

Supporting Information is available from the Wiley Online Library or from the author.

Acknowledgements: M.V.D. and M.S. contributed equally to this work. Generous financial support by the Swiss National Science Foundation (projects 200021_140693, PP00P2_123373, PP00P2_144697, and the National Centre of Competence in Research Bio-Inspired Materials), the Holcim Stiftung Wissen, the Marie Curie Actions of the European Commission, the Swiss Nanoscience Institute, and the Dr. Alfred Bretscher Fund is acknowledged. The authors thank Gabriele Persy and the University of Basel Microscopy Center (ZMB) for the TEM images. Access to cryo-TEM was kindly provided by the Microscopy Imaging Centre of the University of Bern.

Received: November 7, 2014; Revised: December 16, 2014; Published online: January 23, 2015; DOI: 10.1002/marc.201400642

Keywords: amphiphilic block copolymer vesicles; atom transfer radical polymerization (ATRP); biocatalysis; bio-inspiration; nanoreactors

[1] a) P. Tanner, P. Baumann, R. Enea, O. Onaca, C. Palivan, W. Meier, Acc. Chem. Res. 2011, 44, 1039; b) G. Gunkel-Grabole, S. Sigg, M. Lomora, S. Lörcher, C. G. Palivan, W. P. Meier, Biomater. Sci. 2015, 3, 25.

[2] S. F. M. van Dongen, H.-P. M. de Hoog, R. J. R. W. Peters, M. Nallani, R. J. M. Nolte, J. C. M. van Hest, Chem. Rev. 2009, 109, 6212 .

[3] a) K. Renggli, P. Baumann, K. Langowska, O. Onaca, N. Bruns, W. Meier, Adv. Funct. Mater. 2011, 21, 1241; b) R. J. R. W. Peters, I. Louzao, J. C. M. van Hest, Chem. Sci. 2012, 3, 335.

[4] M. Marguet, C. Bonduelle, S. Lecommandoux, Chem. Soc. Rev. 2013, 42, 512.

[5] M. Li, X. Huang, T. Y. D. Tang, S. Mann, Curr. Opin. Chem. Biol. 2014, 22, 1.

[6] a) J. Gaspard, M. S. Hahn, J. A. Silas, Langmuir 2009, 25, 12878 ; b) C. C. Campillo, A. P. Schroder, C. M. Marques, B. Pepin-Donat, Mater. Sci. Eng. C 2009, 29, 393; c) C. Campillo, B. Pepin-Donat, A. Viallat, Soft Matter 2007, 3, 1421; d) M. Faivre, C. Campillo, B. Pepin-Donat, A. Viallat, Prog. Colloid Polym. Sci. 2006, 133, 41.

[7] O. Stauch, T. Uhlmann, M. Fröhlich, R. Thomann, M. El-Badry, Y.-K. Kim, R. Schubert, Biomacromolecules 2002, 3,324 .

[8] a) A. P. Minton, J. Cell Sci. 2006, 119, 2863; b) C. Tan, S. Saurabh, M. P. Bruchez, R. Schwartz, P. LeDuc, Nat. Nanotechnol. 2013, 8, 602 .

[9] a) A. Jesorka, M. Markstroem, O. Orwar, Langmuir 2005, 21, 1230; b) J. S. Lee, W. Zhou, F. Meng, D. Zhang, C. Otto, J. Feijen, J. Controlled Release 2010, 146, 400.

[10] S.-H. Kim, J. W. Kim, D.-H. Kim, S.-H. Han, D. A. Weitz, Small 2013, 9, 124

[11] a) A. M. Smith, M. R. Jaime-Fonseca, L. M. Grover, S. Bakalis, J. Agric. Food Chem. 2010, 58, 4719; b) M. Marguet, O. Sandre, S. Lecommandoux, Langmuir 2012, 28, 2035.

[12] M. Nallani, H. P. M. de Hoog, J. Cornelissen, A. R. A. Palmans, J. C. M. van Hest, R. J. M. Nolte, Biomacromolecules 2007, 8, 3723.

[13] a) O. Stauch, R. Schubert, G. Savin, W. Burchard, Biomacromolecules 2002, 3, 565; b) A. Viallat, J. Dalous, M. Abkarian, Biophys. J. 2004, 86, 2179.

[14] C. Nardin, S. Thoeni, J. Widmer, M. Winterhalter, W. Meier, Chem. Commun. 2000, 1433.

[15] a) M. Spulber, A. Najer, K. Winkelbach, O. Glaied, M. Waser, U. Pieles, W. Meier, N. Bruns, J. Am. Chem. Soc. 2013, 135, 9204; b) J. Gaitzsch, D. Appelhans, L. Wang, G. Battaglia, B. Voit, Angew. Chem. Int. Ed. 2012, 51, 4448.

[16] a) P. B. Zetterlund, Polym. Chem. 2011, 2, 534; b) M. J. Monteiro, M. F. Cunningham, Macromolecules 2012, 45, 4939; c) S. Abe, K. Hirata, T. Ueno, K. Morino, N. Shimizu, M. Yamamoto, M. Takata, E. Yashima, Y. Watanabe, J. Am. Chem. Soc. 2009, 131, 6958; d) K. Renggli, M. G. Nussbaumer, R. Urbani, T. Pfohl, N. Bruns, Angew. Chem. Int. Ed. 2014, 53, 1443; e) S. Deng, J. Zhi, X. Zhang, O. Wu, Y. Ding, A. Hu, Angew. Chem. Int. Ed. 2014, 53, 14144.

[17] a) W. A. Braunecker, K. Matyjaszewski, Prog. Polym. Sci. 2007, 32, 93; b) M. Ouchi, T. Terashima, M. Sawamoto, Chem. Rev. 2009, 109, 4963; c) K. Matyjaszewski, Macromolecules 2012, 45, 4015; d) K. Matyjaszewski, N. V. Tsarevsky, J. Am. Chem. Soc. 2014, 136, 6513 .

[18] a) S. M. Kuiper, M. Nallani, D. M. Vriezema, J. J. L. M. Cornelissen, H. J. C. M. van, R. J. M. Nolte, A. E. Rowan, Org. Biomol. Chem. 2008, 6, 4315; b) F. Axthelm, O. Casse, W. H. Koppenol, T. Nauser, W. Meier, C. G. Palivan, J. Phys. Chem. B 2008, 112, 8211.

[19] S. J. Sigg, F. Seidi, K. Renggli, T. B. Silva, G. Kali, N. Bruns, Macromol. Rapid Commun. 2011, 32, 1710. 
[20] T. B. Silva, M. Spulber, M. K. Kocik, F. Seidi, H. Charan, M. Rother, S. J. Sigg, K. Renggli, G. Kali, N. Bruns, Biomacromolecules 2013, 14, 2703.

[21] a) Y.-H. Ng, F. di Lena, C. L. L. Chai, Chem. Commun. 2011, 47, 6464; b) Y.-H. Ng, F. di Lena, C. L. L. Chai, Polym. Chem. 2011, 2, 589; c) A. Simakova, M. Mackenzie, S. E. Averick, S. Park, K. Matyjaszewski, Angew. Chem. Int. Ed. 2013, 52, 12148; d) G. Gao, M. A. Karaaslan, J. F. Kadla, F. Ko, Green Chem. 2014, 16, 3890.

[22] D. Wu, M. Spulber, F. Itel, M. Chami, T. Pfohl, C. G. Palivan, W. Meier, Macromolecules 2014, 47, 5060.
[23] C. Nardin, T. Hirt, J. Leukel, W. Meier, Langmuir 2000, 16, 1035.

[24] a) W. Burchard, in Physical Techniques for the Study of Food Biopolymers (Ed: S. B. Ross-Murphy), Blackie Academics and Professional, New York, NY, USA 1994, p. 151; b) W. Burchard, in Light Scattering from Polymers, Adv. Polymer Sci., Vol. 48, Springer, Berlin, Heidelberg 1983, p. 1.

[25] F. Itel, M. Chami, A. Najer, S. Lörcher, D. Wu, I. A. Dinu, W. Meier, Macromolecules 2014, 47, 7588.

[26] G. Battaglia, A. J. Ryan, J. Phys. Chem. B 2006, 110, 10272.

[27] P. Tanner, O. Onaca, V. Balasubramanian, W. Meier, C. G. Palivan, Chem. Eur. J. 2011, 17, 4552. 